\title{
Propriedades elásticas e elétricas de rochas estimadas computacionalmente
}

\author{
Lizianne Carvalho Medeiros ${ }^{1}$, José Agnelo Soares ${ }^{1}$, Rodrigo Surmas ${ }^{2}$, Celso Peres Fernandes ${ }^{3}$ \\ ${ }^{1}$ Universidade Federal de Campina Grande, ${ }^{2}$ PETROBRAS/CENPES, ${ }^{3}$ Universidade Federal de Santa Catarina
}

\section{Copyright 2014, SBGf - Sociedade Brasileira de Geofísica}

Este texto foi preparado para a apresentação no VI Simpósio Brasileiro de Geofísica, Porto Alegre, 14 a 16 de outubro de 2014. Seu conteúdo foi revisado pelo Comitê Técnico do VI SimBGt, mas não necessariamente representa a opinião da SBGf ou de seus associados. É proibida a reprodução total ou parcial deste material para propósitos comerciais sem prévia autorização da SBGt.

\section{Resumo}

É realizada neste trabalho a estimativa das propriedades petrofísicas de amostras de rochas a partir de imagens de microtomografia de raios $x$. São demonstrados os procedimentos adotados, os resultados obtidos e realizada uma comparação das propriedades simuladas computacionalmente com os valores medidos através de métodos convencionais de laboratório. A simulação da velocidade de propagação da onda $P$ foi realizada com sucesso para modelos $3 D$ de subamostras de arenito. Os resultados obtidos para o fator de formação se mostraram favoráveis para subamostras homogêneas de arenito e carbonato. É realizada uma análise da influência de argilominerais e da microporosidade presentes nos modelos virtuais de rocha, para aos valores simulados do fator de formação.

\section{Introdução}

Convencionalmente as propriedades petrofísicas essenciais para a caracterização dos reservatórios quanto à sua capacidade de armazenamento e de produtividade de fluidos - são determinadas experimentalmente através de ensaios físicos em laboratório. Esses ensaios são realizados principalmente em plugues extraídos de testemunhos de poços, os quais possuem dimensões centimétricas.

As propriedades petrofísicas assim determinadas precisam ser agrupadas a fim de representarem o comportamento em uma escala maior, como a do reservatório. Para a realização de ensaios laboratoriais representativos das condições in situ do reservatório diversos desafios devem ser equacionados, como por exemplo, as condições de pressão, temperatura e saturação fluida a serem adotadas nos ensaios, o tempo que os plugues devem ser mantidos sob essas condições de modo a terem seu comportamento in situ restabelecido e garantir a preservação dos plugues após a sua extração. Além desses e de outros desafios, nem sempre é possível reproduzir fisicamente uma condição específica, como realizar ensaios físicos sob saturação com óleo vivo, sob altas pressões e, simultaneamente, sob altas temperaturas.

Recentemente diversos autores (Knackstedt et al., 2009; Dvorkin et al., 2011; Zhang et al., 2011; Medeiros et al., 2012) tem demonstrado a possibilidade de estimar as propriedades petrofísicas a partir da análise computacional de imagens de rochas obtidas por microtomografia de raios $x$. As vantagens desta técnica dizem respeito à sua capacidade de quantificar as propriedades físicas das rochas sob praticamente qualquer condição imaginável, para um grande número de subamostras, de forma rápida, com ensaios que independem da ordem de execução e que podem ser reproduzidos a qualquer tempo. Para isso é preciso dispor de imagens com resolução suficiente para reconhecer e quantificar as estruturas que controlam o comportamento físico das rochas.

As propriedades petrofísicas que são estimadas neste trabalho a partir de imagens microtomográficas são porosidade, permeabilidade absoluta, velocidade de propagação da onda $\mathrm{P}$ e propriedades elétricas. $\mathrm{O}$ objetivo deste trabalho é apresentar resultados comparativos entre estas propriedades obtidas em ensaios físicos e em análises computacionais.

\section{Metodologia}

São medidas ou estimadas neste trabalho a porosidade, a permeabilidade absoluta, a velocidade da onda $P$ para quatro plugues de arenito (A4, A7, A9) e um de folhelho (F9) oriundos de um furo estratigráfico na Bacia do Rio do peixe (BRP), e o fator de formação para plugues de arenito e carbonato, oriundos de afloramentos, disponibilizados pela PETROBRAS.

Neste trabalho foram utilizados métodos de injeção gasosa nos plugues para a medição laboratorial da porosidade e da permeabilidade, utilizando o UltraPoreperm $500^{\circledR}$ fabricado pela Core Lab Instruments e disponível no Laboratório de Petrofísica da UFCG. O método de medição de porosidade é baseado na lei fundamental dos gases de Boyle-Mariotte, a qual se baseia na variação de volume e pressão de um gás ideal em temperatura constante. A permeabilidade é medida através da aplicação da equação de Darcy, que diz que a perda de pressão que um fluido sofre ao atravessar um meio poroso é diretamente proporcional à vazão desse fluido.

Para as medições das velocidades de propagação das ondas $\mathrm{P}$ em amostras secas e submetidas a pressões confinantes de 5 a $40 \mathrm{MPa}$, sob temperatura ambiente foi utilizado o sistema Autolab $500^{\circledR}$, fornecido pela New England Research e também disponível no Laboratório de Petrofísica da UFCG.

As propriedades elétricas de rochas são determinadas através de ensaios físicos em laboratório, e fornecem parâmetros como o fator de formação (FF) e o índice de resistividade $(n)$, os quais são utilizados para a estimativa do volume de hidrocarbonetos presente no reservatório. O fator de formação é a razão entre a resistividade elétrica da rocha saturada por um fluido condutor e a resistividade elétrica deste fluido. $O$ fator de formação está ainda relacionado à porosidade da rocha, segundo a Lei de Archie (1942). Um fator determinante para a resistividade da rocha é a presença de argilas, pois a presença deste mineral tem dois efeitos, no que diz respeito ao seu comportamento geoelétrico: por um lado diminui a resistividade elétrica do material e, por outro, torna essa propriedade dependente da frequência do campo eletromagnético. 
Nas rochas carbonáticas a presença de argilominerais é menos comum do que nas rochas siliciclásticas, embora seja possível a sua ocorrência em litotipos específicos como em tufas carbonáticas e em margas. Para rochas carbonáticas, a resistividade sofre uma maior variação em função da microporosidade existente nestas rochas.

Os resultados dos ensaios laboratoriais das propriedades elétricas dos plugues de arenito e de carbonato foram fornecidos pela PETROBRAS.

Para a realização do imageamento por microtomografia de raios $\mathrm{x}$ foram extraídas subamostras, cortadas da extremidade superior dos plugues utilizados nos ensaios convencionais (Figura 1). As subamostras apresentam a forma de pequenos paralelepípedos com dimensões aproximadas de $10 \mathrm{~mm} \times 8 \mathrm{~mm} \times 7 \mathrm{~mm}$ ou de cilindros com $7 \mathrm{~mm}$ de diâmetro e $10 \mathrm{~mm}$ de comprimento.
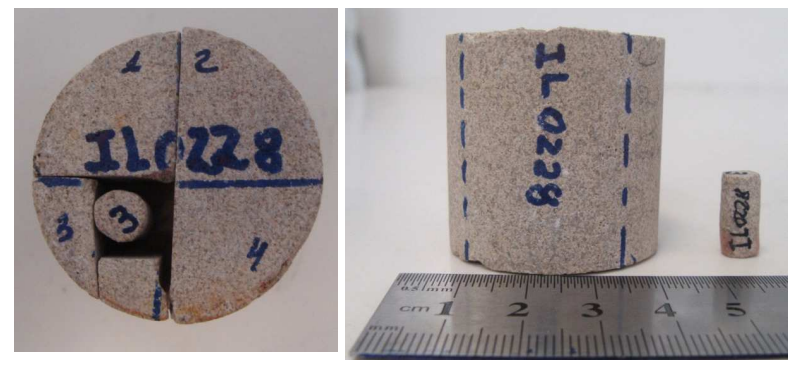

Figura 1 - Subamostra extraída da extremidade superior do plugue.

As aquisições microtomográficas foram realizadas no Laboratório de Meios Porosos e Propriedades Termofísicas da Universidade Federal de Santa Catarina (amostras para cálculo de porosidade, permeabilidade absoluta e velocidade acústica) e no Centro de Pesquisas da PETROBRAS (amostras para estimativa das propriedades elétricas).

A microtomografia computadorizada é realizada com exposições radiográficas sequenciais utilizando uma fonte de raios $\mathrm{x}$, com um detector posicionado atrás do objeto, o qual é rotacionado a cada exposição até que toda a área de interesse seja radiografada. Os dados do microtomógrafo são registrados em diversos ângulos na forma de projeções do objeto, obtendo as chamadas radiografias. Em seguida esses dados brutos são processados a fim de gerar os tomogramas bidimensionais (slices), os quais quando empilhados, geram uma imagem tridimensional do objeto imageado (Figura 2).

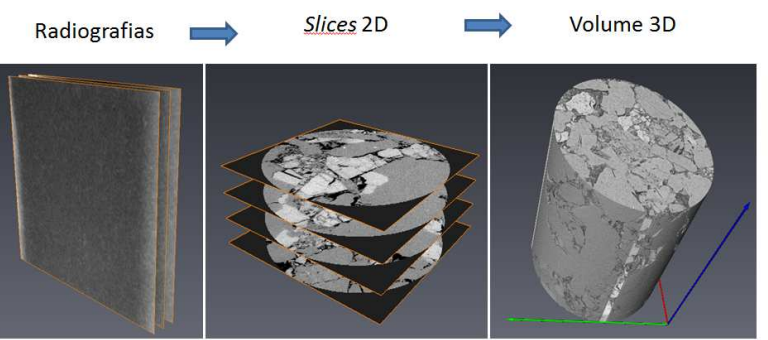

Figura 2 - Conjunto de radiografias, slices horizontais e reconstrução $3 D$ de uma subamostra.

Cada subamostra é composta por mais de 1500 slices, sendo que cada slice é composto por cerca de 1,4 MB.
Por esta razão a simulação das propriedades petrofísicas de uma subamostra pode se constituir em uma tarefa que exige muitos recursos computacionais. Dessa forma são utilizados neste trabalho volumes menores, extraídos destas subamostras virtuais.

Para o processamento dos dados, tratamento e segmentação das imagens, geração de malhas tetraédricas, estimação da porosidade e simulação da permeabilidade absoluta é utilizado o software Avizo Fire $^{\circledR}$ 8. Para a simulação da propagação da onda $P$ utiliza-se o Comsol Multiphysics ${ }^{\circledR} 4.3$, o qual é baseado no método de elementos finitos, e para a determinação do fator de formação é utilizado o software Morphy ${ }^{\circledR}$, desenvolvido pela Universidade Nacional da Austrália.

Através das imagens microtomográficas é possível visualizar e quantificar o espaço poroso. A Figura 3 apresenta um histograma de tons de cinza dos pixels das imagens, obtido utilizando o software Avizo ${ }^{\circledR}$. O histograma traça a frequência ou o número de ocorrências de pixels com mesma escala de cinza, assim é possível através deste dividir as fases existentes na imagem, delimitando um limiar entre os tons de cinza que representam o espaço poroso (tons de cinza mais escuros) e os tons de cinza que representam a matriz mineral (tons de cinza claros).

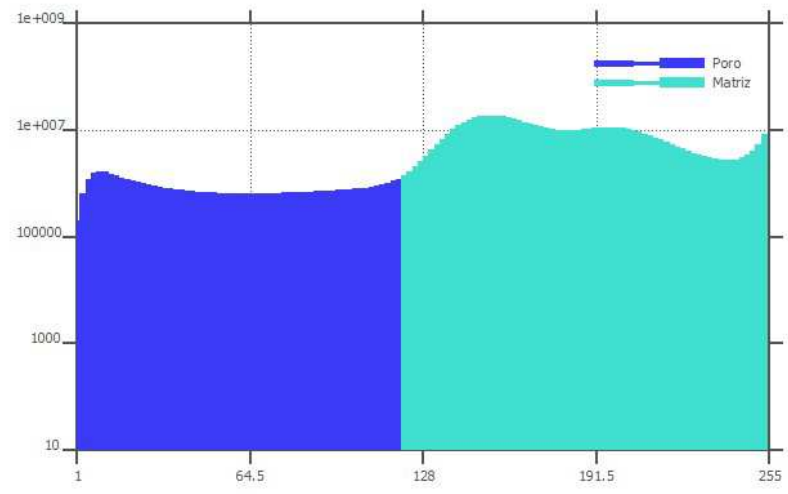

Figura 3 - Histograma representando os níveis de tons de cinza das imagens.

A partir do histograma foi estabelecido o limiar utilizado para a segmentação das imagens. A Figura 4 apresenta um exemplo de imagem 2D e 3D segmentados.

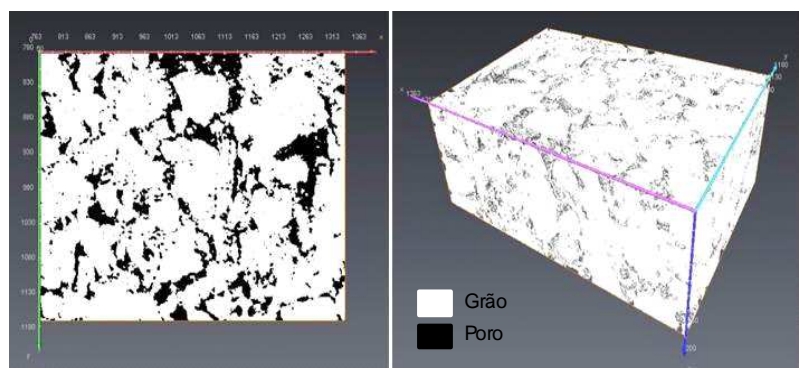

Figura 4 - Imagens 2D e 3D obtidas após a segmentação.

O Avizo ${ }^{\circledR}$ dispõe de uma ferramenta para medição do volume de material existente na imagem, o Material Statistics. Através dessa ferramenta é possível obter o volume do espaço poroso (pixels mais escuros que o limiar) e o volume da matriz mineral (pixels mais claros 
que o limiar), podendo então ser calculada a porosidade das subamostras.

O cálculo da porosidade para as subamostras que apresentam poros abaixo da resolução do estudo é baseado no procedimento acima citado e em uma análise do valor da microporosidade, a qual é calculada também no Avizo ${ }^{\circledR}$ a partir dos valores dos coeficientes de atenuação ou limiar das imagens.

Primeiramente é determinado o limiar da fase poro, da fase sólida e da fase intermediária que contêm os microporos. Posteriormente é realizado um cálculo para obter o valor da porosidade existente nessa fase intermediária.

A permeabilidade absoluta do meio poroso, a partir do conjunto de imagens microtomográficas, é estimada através de uma simulação numérica baseada na solução da equação de Navier-Stokes. Após a segmentação do subvolume aplica-se a ferramenta $X$ Lab Hydro, e configura as condições de contorno, a direção do fluxo e a viscosidade do fluido que serão adotados na simulação. Os dados de saída desta ferramenta são campos de pressão e/ou velocidade de fluxo do fluido e os valores de permeabilidade absoluta. A Figura 5 mostra as linhas de fluxo para a simulação da permeabilidade absoluta e as condições de contorno adotadas para este trabalho.

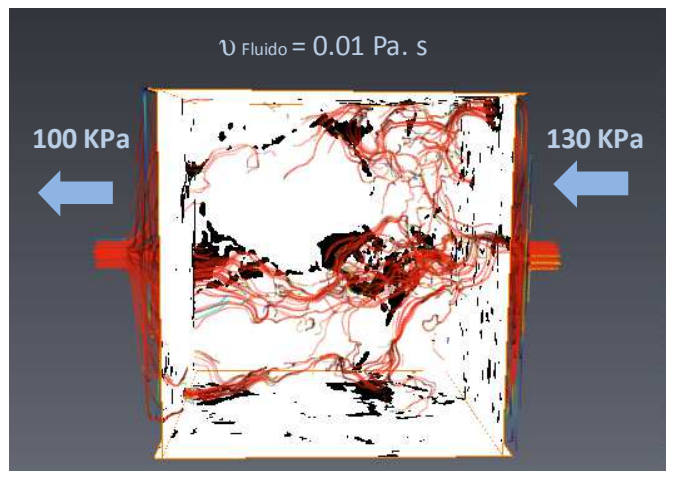

Figura 5 - Linhas de fluxo e condições de contorno para a simulação da permeabilidade.

Devido a limitações computacionais cada subamostra foi dividida em ROls (Regions of Interest). Em seguida a permeabilidade de cada ROI foi simulada, sendo a permeabilidade da subamostra estimada como a média das permeabilidades dos ROls que pertencem aquela subamostra. Embora Tiab \& Donaldson (2004) recomendem o uso da média geométrica para o cálculo da permeabilidade a partir de um conjunto de valores simulados, neste trabalho observou-se que a média aritmética dos ROls se aproxima melhor dos valores de permeabilidade medidos fisicamente. Portanto, esta foi a média adotada neste trabalho.

Neste trabalho a obtenção da velocidade de propagação da onda compressional a partir das imagens microtomográficas é baseada em uma modelagem 3D. É necessário primeiramente gerar uma malha tetraédrica no Avizo ${ }^{\circledR}$ e posteriormente exportála para o $\mathrm{Comsol}^{\circledR}$, onde será construído o modelo para a propagação de onda $P$ com 0 módulo de Pressão Acústica Transiente. Na Figura 6 é interessante observar a distribuição dos elementos tetraedrais na malha e como ela se adapta às heterogeneidades do domínio, de modo que os elementos são maiores dentro dos grãos minerais de maiores dimensões e menores nos poros e nos grãos menores.

Inicialmente os algoritmos da modelagem foram aplicados para o caso de um domínio 3D de alumínio. Isto foi feito como uma forma de calibrar o modelo de simulação da onda compressional em um domínio de propriedades elásticas simples e previamente conhecidas.

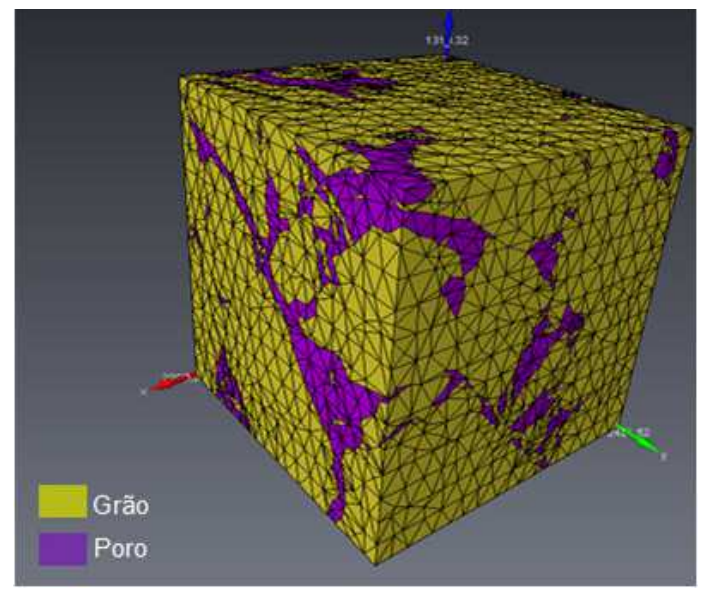

Figura 6- Malha adaptativa de elementos finitos utilizada na modelagem de propagação da onda $P$.

Para a propagação da onda $\mathrm{P}$ no volume de alumínio foi adotada na extremidade superior do domínio uma pressão gerada a partir de uma fonte de onda, oscilando em uma frequência de $1 \mathrm{MHz}$ e amplitude de $100 \mathrm{~Pa}$ durante $1 \mu \mathrm{s}$. A malha adaptativa adotada é apresentada na Figura 7.

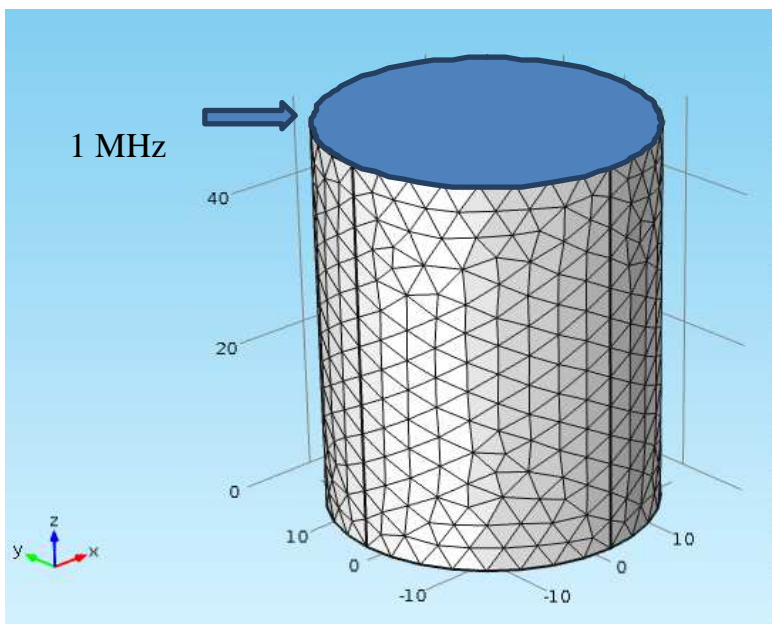

Figura 7 - Malha tetraédrica utilizada na modelagem de propagação da onda $P$ no plugue virtual de alumínio.

Após a calibração do algoritmo foi construído um modelo 3D para a simulação com as subamostras. Considera-se no modelo 3D a matriz mineral composta por arenito com densidade de $2300 \mathrm{Kg} / \mathrm{m}^{3}$ e velocidade de propagação da onda $P$ de $4000 \mathrm{~m} / \mathrm{s}$ e poros contendo argila com densidade de $2000 \mathrm{Kg} / \mathrm{m}^{3} \mathrm{e}$ velocidade de propagação da onda P de $2500 \mathrm{~m} / \mathrm{s}$. 
Para o início das simulações do fator de formação com - Morphy ${ }^{\circledR}$, as imagens foram segmentadas considerando a fase poro, a fase sólida, e a microporosidade para amostra com poros sob resolução. Neste estudo as subamostras possuíam $800^{3}$ slices e são cortados em subvolumes de $400^{3}$ e $200^{3}$ slices, para que seja observada a variação dos valores do fator de formação em função da variação do volume da subamostra analisada.

A simulação é então realizada para todos os subvolumes $\left(800^{3}, 400^{3}\right.$ e $\left.200^{3}\right)$ da subamostra e 0 fator de formação é calculado considerando que a condutividade da matriz mineral é igual a zero e que o espaço poroso está preenchido por um fluido condutor. Para a fase contendo a microporosidade foram adotados valores de condutividade em função da porosidade estimada para o subvolume.

\section{Resultados}

A Figura 8 apresenta uma comparação entre os valores de porosidade medidos nos plugues e os valores de porosidade estimados através da simulação computacional para as subamostras dos plugues de arenito (A4, A7, A9) e folhelho (F9) oriundos da BRP. Vê-se nesta figura que os valores de porosidade estimados a partir das imagens são aproximadamente iguais aos valores de porosidade medidos nos ensaios convencionais, pois se a correspondência fosse perfeita, os pontos da figura cairiam sobre a linha diagonal positiva do gráfico. Isto indica que a resolução de $8 \mu \mathrm{m}$ para as subamostras A7 e A4 é suficiente para obter estimativas corretas de porosidade através da simulação computacional. O desvio observado para as amostras de granulometria mais fina, como o folhelho, ocorre porque a resolução não é suficiente para o reconhecimento completo dos poros e gargantas de poro de menores dimensões, mesmo que seja realizada a análise da microposidade.

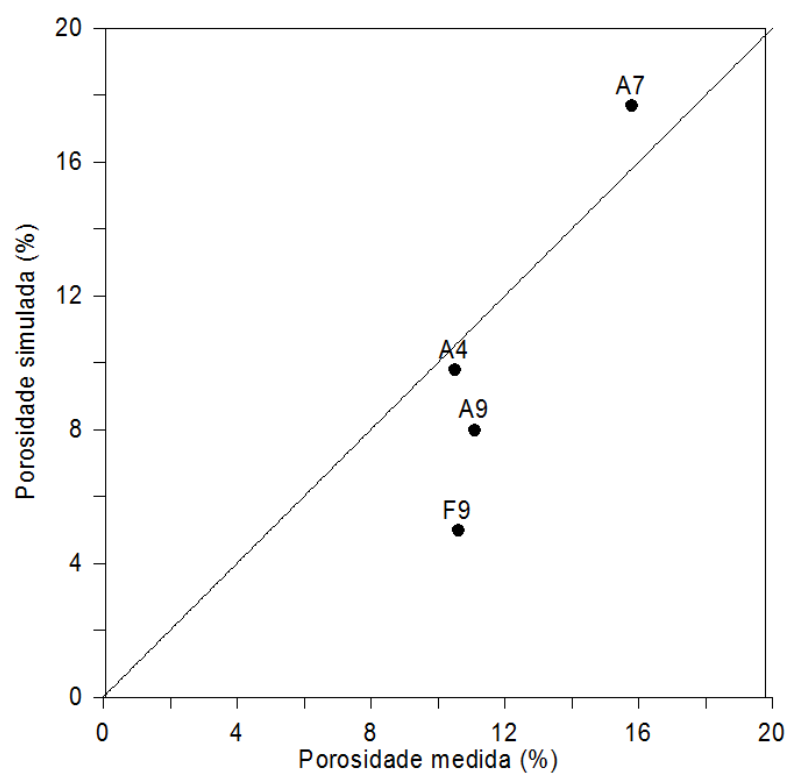

Figura 8 - Comparação entre os valores de porosidade estimados na simulação (subamostras) e os obtidos nos ensaios convencionais (plugues).
A Figura 9 apresenta os resultados obtidos na simulação da permeabilidade das quatro subamostras em comparação com os respectivos valores de permeabilidade medidos fisicamente através do fluxo de gás nos plugues em laboratório. Desta figura podese ver que os valores de permeabilidade estimados se encontram na mesma ordem de grandeza dos valores medidos nas amostras de granulometria mais grossa (A7 e A4), mas apresentam uma discrepância para as amostras de granulometria mais fina (A9 e F9). Devese ressaltar que, devido a limitações computacionais, os valores de permeabilidade foram simulados para ROls e calculada a média para as subamostras, enquanto que os ensaios físicos foram realizados em plugues.

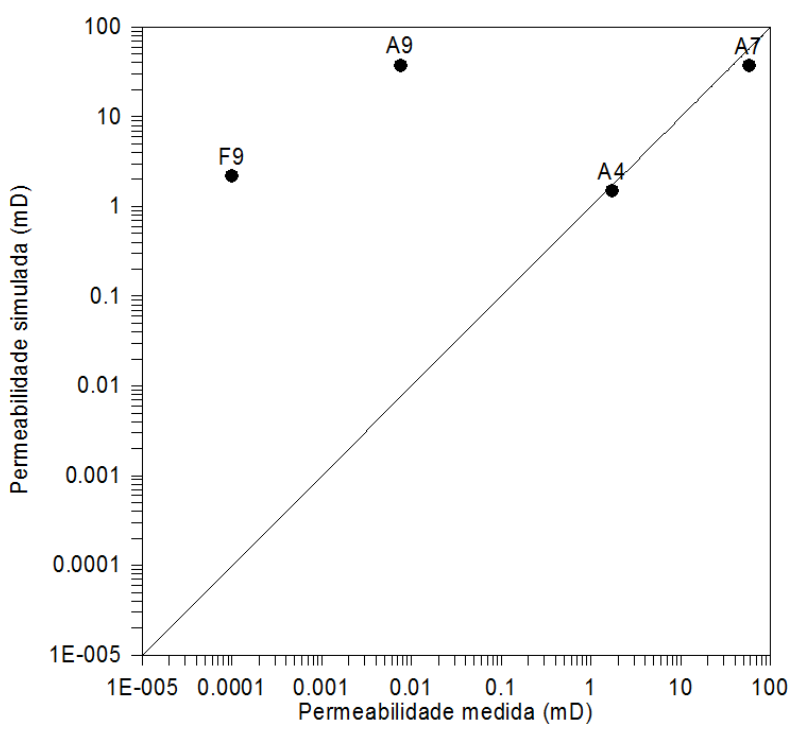

Figura 9- Comparação entre os valores de permeabilidade simulados (subamostras) e os obtidos nos ensaios convencionais (plugues).

A Figura 10 apresenta quatro snapshots que representam momentos distintos durante a simulação numérica da propagação da onda $P$ no volume $3 D$ de alumínio. Considerando que a velocidade da onda $\mathrm{P}$ no alumínio é de $6300 \mathrm{~m} / \mathrm{s}$ e que o comprimento do cilindro é de $5 \mathrm{~cm}$, o tempo necessário para a onda atingir a extremidade inferior do cilindro é de aproximadamente $8 \mu \mathrm{s}$. Nesta figura o snapshot "d" representa a frente de onda para o tempo $t=7,5 \mu \mathrm{s}$, portanto, em um tempo imediatamente anterior à chegada da onda na extremidade inferior do modelo. Isto comprova a eficiência do modelo utilizado para a simulação da propagação da onda $P$ e calibra esta metodologia antes de sua aplicação para o caso de rochas. 


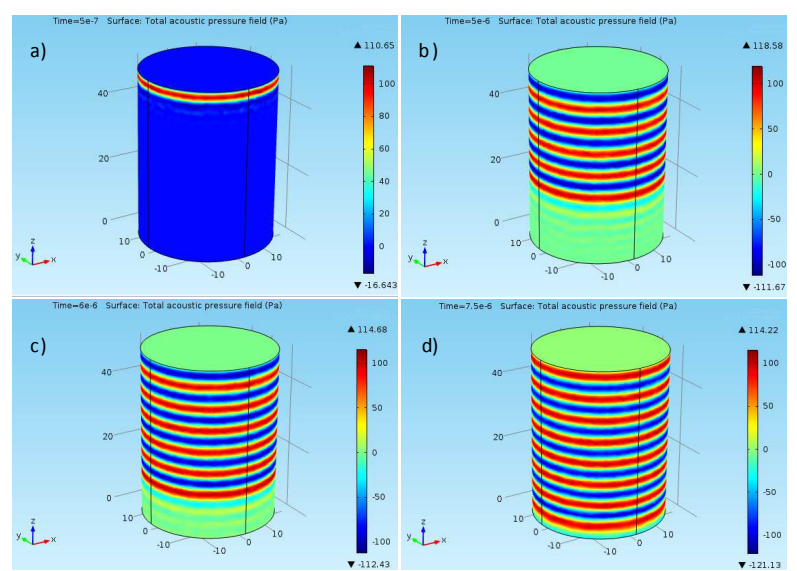

Figura 10 - Snapshots da modelagem da propagação da onda $P$ no cilindro de alumínio em quatro momentos: a) $t=0,5 \mu \mathrm{s}$; b) $t=5 \mu \mathrm{s}$; c) $t=6 \mu \mathrm{s}$ e d) $t=$ $7,5 \mu \mathrm{s}$.

De modo semelhante ao que foi apresentado para o caso do plugue virtual de alumínio, a Figura 11 apresenta uma sequência de snapshots que representa a evolução da frente de onda compressional para a subamostra A4 em quatro momentos distintos: a) $\mathrm{t}=$ $0,02 \mu \mathrm{s}$; b) $\mathrm{t}=0,1 \mu \mathrm{s}$; c) $\mathrm{t}=0,15 \mu \mathrm{s}$ e d) $\mathrm{t}=0,19 \mu \mathrm{s}$.

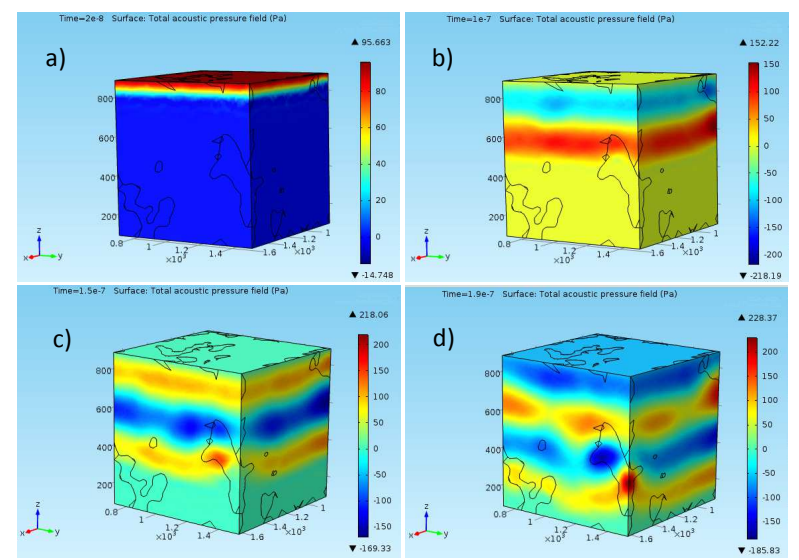

Figura 11 - Snapshots da onda $P$ em um subvolume de uma subamostra em quatro momentos: a) $t=0,02 \mu \mathrm{s}$; b) $t=0,1 \mu \mathrm{s}$; c) $t=0,15 \mu \mathrm{s}$ e d) $t=0,19 \mu \mathrm{s}$.

O traço acústico resultante da modelagem da propagação da onda em um ponto localizado no centro da extremidade inferior do subvolume é apresentado na Figura 12. Pode-se observar que o tempo de chegada da onda (primeira quebra) é de aproximadamente $0,2 \mu \mathrm{s}$, o que é coerente com 0 snapshot "d" da Figura 11. Como o volume 3D possui comprimento de $793 \mu \mathrm{m}$, a velocidade de propagação da onda $P$ no subvolume é de $3900 \mathrm{~m} / \mathrm{s}$.

$\mathrm{Na}$ Tabela 1 são observados os valores obtidos na simulação da propagação da onda $P$ para as subamostras dos arenitos da BRP.

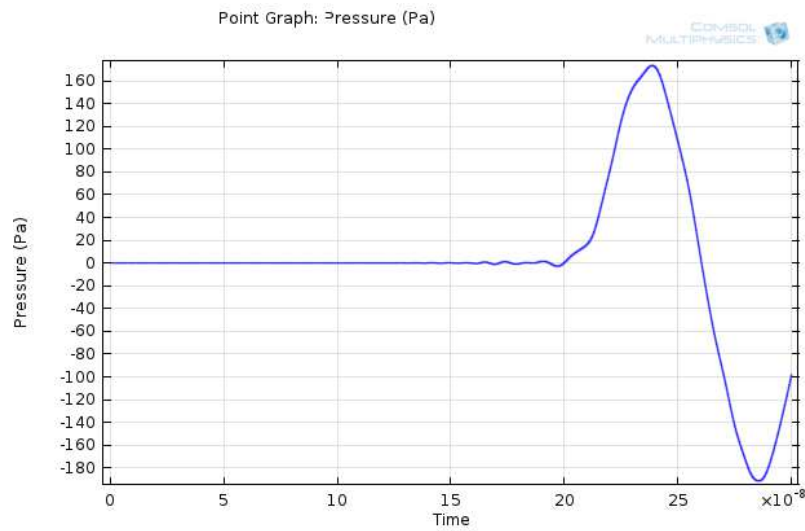

Figura 12 - Traço acústico simulado para um ponto localizado no centro da extremidade inferior de um slice da subamostra de arenito A4.

Tabela 1 - Valores de velocidade simulados para a propagação da onda $P$.

\begin{tabular}{|c|c|}
\hline Subamostra & VP $(\mathrm{m} / \mathrm{s})$ \\
\hline A4 & 3750 \\
\hline A7 & 3900 \\
\hline A9 & 3520 \\
\hline
\end{tabular}

Considerando que todos os modelos $3 D$ das subamostras de arenito se constituem em um domínio heterogêneo cujas fases apresentam velocidades individuais de $4000 \mathrm{~m} / \mathrm{s}$ (arenito) e $2500 \mathrm{~m} / \mathrm{s}$ (argila), as velocidades simuladas se encontram dentro do intervalo esperado.

O fator de formação simulado para as subamostras dos plugues de arenito, apesar da diferença de escala, se aproxima do resultado de laboratório e apresenta uma tendência. Nota-se também que o fator de cimentação (m) está entre 1,91, obtido através do resultado de laboratório, e 1,75, para a subamostra do arenito IBS0228sub4 (Figura 13).

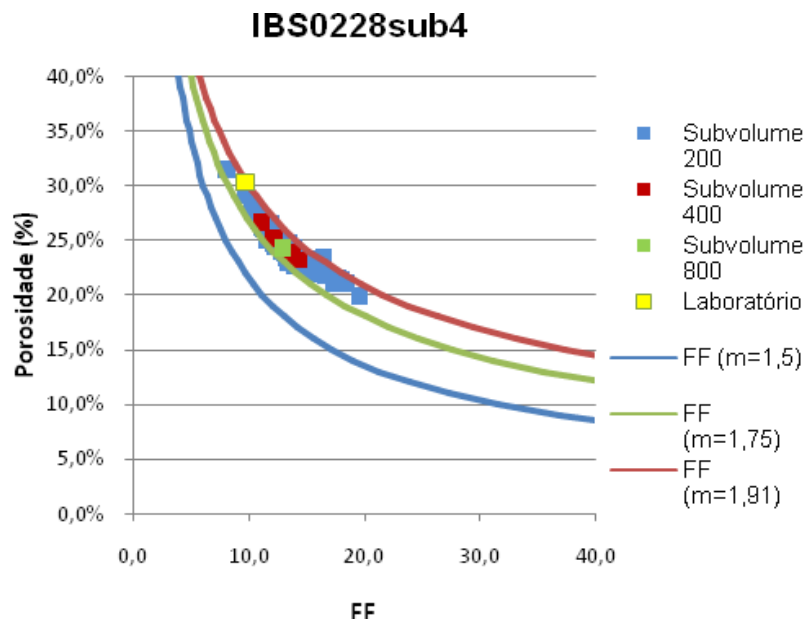

Figura 13 - Comparação entre os valores do fator de Formação (FF) obtido nas simulações para a subamostra IBS0228sub4 sem a presença da argila e o valor obtido em laboratório. 
Para os carbonatos os valores simulados do fator de formação e da porosidade para os subvolumes se mostraram eficientes em subamostras mais homogêneas como é o caso da IL0228 (Figura14).

\section{IL0228}

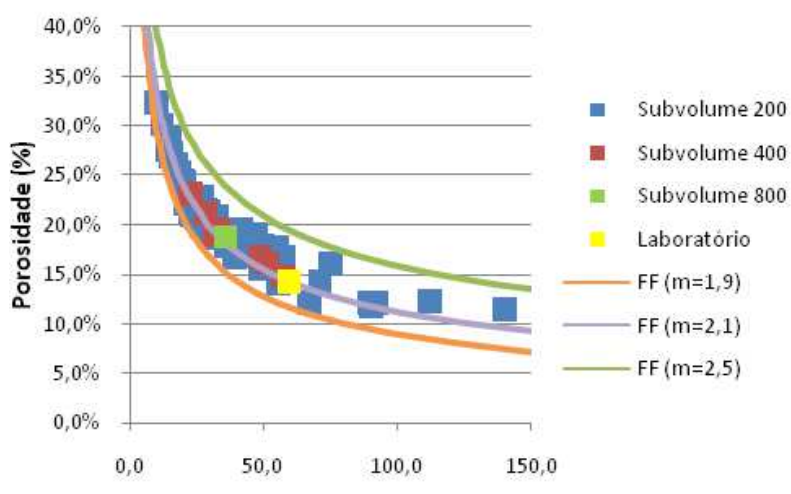

FF

Figura 14 - Comparação entre os resultados do fator de formação estimados nas simulações para a subamostra IL0228 e o resultado obtido em laboratório.

Para subamostras do carbonato SD0214, observa-se uma divergência dos valores obtidos na simulação em relação aos valores de laboratório (Figura 15). Essa amostra possui uma estrutura bastante heterogênea que pode ser observada em várias escalas, dificultando assim a escolha do volume representativo para 0 processo da simulação.

\section{SD0214}

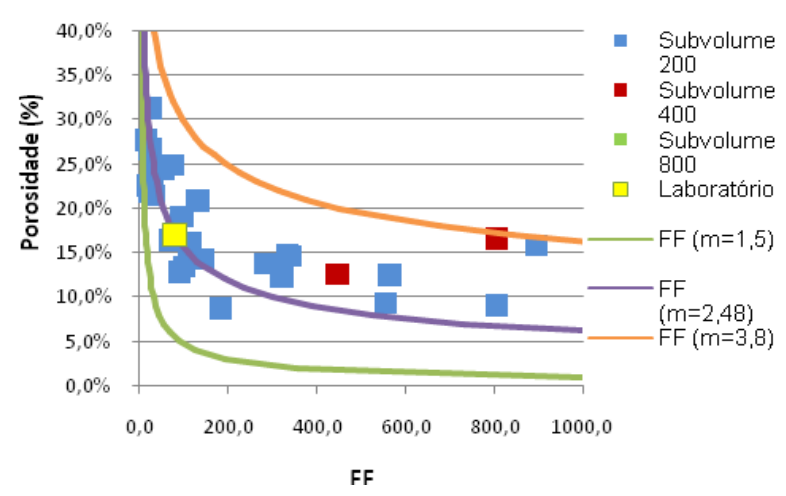

Figura 15 - Resultados do fator de formação estimados nas simulações para a subamostra SD0214 e o resultado obtido em laboratório

\section{Conclusões}

Este trabalho demonstra a viabilidade da determinação das propriedades permoporosas, acústicas e elétricas de rochas a partir de imagens de microtomografia de raios $x$. A resolução das imagens necessária para a correta simulação dessas propriedades depende do tamanho dos grãos e dos poros das rochas analisadas.

As propriedades permoporosas simuladas neste trabalho foram comparadas com medições laboratoriais. Os valores de porosidade estimados por microtomografia foram quase coincidentes com os valores medidos nos ensaios laboratoriais.

Para a simulação da permeabilidade observou-se que os valores aproximadamente coincidem com as permeabilidades medidas nos ensaios convencionais, especialmente para as amostras de granulometria não muito fina. Isto indica a necessidade de resoluções das imagens ainda mais altas, da ordem de nanômetros para a simulação precisa da permeabilidade de rochas de granulometria muito fina como siltes e folhelhos.

A modelagem numérica $3 D$ da propagação da onda compressional foi realizada com sucesso para as subamostras de arenitos. Os modelos utilizados foram inicialmente calibrados em um domínio 3D de um plugue de alumínio, o qual apresentou resultados inteiramente compatíveis com as propriedades daquele material.

Os resultados obtidos para a porosidade e para o fator de formação na simulação em arenitos foram bem semelhantes aos resultados de laboratório, demonstrando a importância da análise de microporosidade e a eficiência do software utilizado. Observa-se também que os resultados foram favoráveis independentemente da escala $\left(200^{3}, 400^{3}\right.$, $800^{3}$ ), gerando tendências que permitem o conhecimento da relação entre estas propriedades.

Para as simulações nas subamostras de carbonatos foram obtidos resultados coerentes para as subamostras mais homogêneas, demonstrando a viabilidade das simulações também para este tipo de rocha.

\section{Agradecimentos}

Os autores agradecem aos convênios PETROBRAS/UFCG TC 0050.0057323.10.9 e TC 0050.0078516 .12 .9 pelo financiamento da pesquisa e à PETROBRAS pela permissão para a publicação destes resultados.

\section{Referências}

ARCHIE, G. E. (1942). Electrical resistivity log as an aid in determining some reservoir characteristics. Trans AIME, v. 146, p. 54-67.

DVORKIN, J; DERZHI, N; DIAZ, E; FANG, Q. (2011). Relevance of computational rock physics. Geophysics, Vol.76, n. 5.

KNACKSTEDT, M.A.; LATHAM, S.; MADADI, M.; SHEPPARD, A.; VARSLOT, T.; ARNS, C. (2009). Digital rock physics: 3D imaging of core material and correlations to acoustic and flow properties. The Leading Edge. January.

MEDEIROS, L.C.; SOARES, J.A.; PORTO, A.L. (2012). Permoporosidade computacional de rochas da Bacia do Rio do Peixe. V Simpósio Brasileiro de Geofísica. Salvador, BA.

TIAB, D.; DONALDSON, E.C. (2004). Petrophysics. Theory and practice of measuring reservoir rock and fluid transport properties. Elsevier.

ZHANG, S; SAXENA, S; BARTHELEMY, P; MARSH, M; MAVKO, G; MUKERJI, T. (2011). Poromechanics investigation at pore-scale using Digital Rock Physics Laboratory. Comsol Conference. 